\title{
LE PLAN D'ÉLECTRIFICATION DU CHILI
}

\author{
J. RICHTERICH \\ Ingénieur aux Ateliers N. B. P. P.
}

L'accueil cordial et intéressé qui m'a été réservé par les Ingénieurs de l'Administration du Chili, lors de ma récente visite à Santiago, l'empressement et l'enthousiasme avec lequel m'ont parlé du problème de l'Electrification de leur pays, MM. Moore et Hardecker, respectivement Directeur Général et Ingénieur en Chef de l'Empresa Nacional de Electricidad, m'encouragent à exposer sommairement l'ampleur de leurs projets. Un tel résumé se justifie d'autant plus que le gouvernement du Chili fait appel, actuellement, pour la réalisation du programme d'équipement qu'il s'est tracé, à la collaboration des constructeurs internationaux, américains et européens.

Le Chili, pays de $751.500 \mathrm{~km}^{2}$ de surface, de forme très allongée de $4.000 \mathrm{~km}$ de longueur et seulement $200 \mathrm{~km}$ de largeur en moyenne, ne possède pas de combustible liquide ef très peu de charbon (réserve de l'ordre de 50 tonnes/ habitant).

La seule richesse d'énergie est l'eau et cette richesse est importante. Pourtant, elle n'a été exploitée que faiblement avant la guerre; c'est ainsi qu'en $1942,3 \%$ seulement de la ressource hydraulique disponible était équipée.

Depuis de nombreuses années, un certain nombre d'Ingénieurs chiliens se sont attachés au problème de l'étude systématique de la réserve hydroélectrique de leur pays, et ont abouti à dresser un inventaire des chutes d'eau possibles très complet et rationnellement présenté. Ce travail particulièrement difficile a été confirmé par une décision administrative, prise le 23 aout 1939, fixant pour la première fois, l'établissement d'un plan d'action immédiat pour l'électrification du Chili. La mise au point d'un tel plan fut confiée à la « Corporacion de Fomento de la Produccion ", organisme d'Etat qui a pour tàche le développement de toutes les activités industrielles du pays.

C'est à partir de cette date qu'a été élaboré le plan définitif d'électrification, allant de pair et s'adaptant parfaitement à des études importantes pour l'industrialisation et le développeinent agricole intensif du pays.

Ce plan comprend une ouvre fondamentale (plan de electrificacion primaria) et des auvres complémentaires.

Le plan fondamental prévoit la construction d'un premier lot de centrales productrices et les lignes de distribution principales. La Corporacion de Fomento reste ainsi propriétaire de la production et de la distribution principale, c'està-dire de la distribution en bloc et la vente de courant aux sociétés de distribution secondaire, aux industries privées ou d'Etat, à des coopératives agricoles, etc. Elle restera ainsi maître des prix de production et de la vente en gros, ce qui lui permet de contrôler également les prix de vente en détail.

Le plan complémentaire prévoit:

- L'irrigation mécanique, développement des irrigations par pompage pour permettre de gagner à grande échelle des terrains non encore exploités, parce que pas arrosables par gravité.

- Un plan d'électrification rurale, sous forme d'une aide administrative financière et technique, à des coopératives agricoles, qui crées sous l'impulsion de l'état, réaliseront elles-mêmes les réseaux de distribution interne et développeront au maximum l'utilisation de courant électrique pour les buts agricoles.

- Un plan d'électrification urbaine, sous forme d'aide technique et financière pour l'amélioration et le développement de réseaux urbains.

- Un plan d'enseignement et de diffusion, dans le but d'augmenter au maximum l'utilisation rationnelle du courant.

Le «Plan de Electrificacion primaria » a éte approuvé le 9 avril 1943 et immédiatement mis en application, sous la forme suivante : 


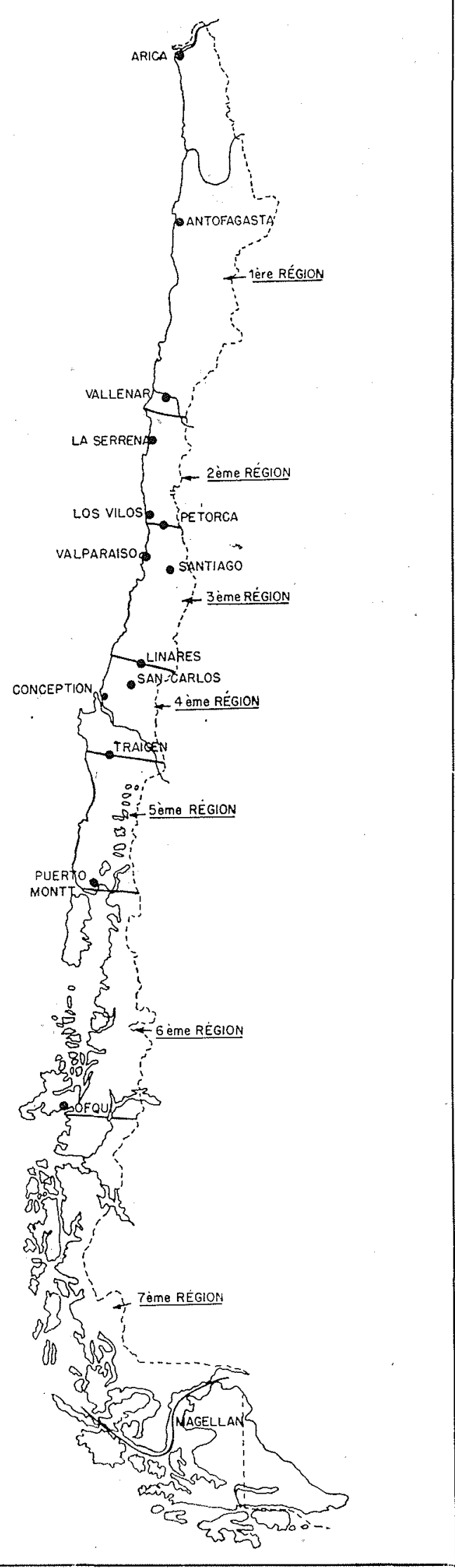

\section{POTENTIEL TOTAL HYDROELECTRIQUE}

L'ensemble du territoire du Chili a été divisé en 7 grandes régions géographiques, chacune définie par un régime hydrologique et des ressources hydro et thermoélectriques distincts, ainsi que par des conditions de répartition el d'utilisation de courant particulières.

Nous avons porté sur le tableau ci-après la répartition de ces zones, leur potentiel hydroélectrique et les caractéristiques du régime hydrologique.

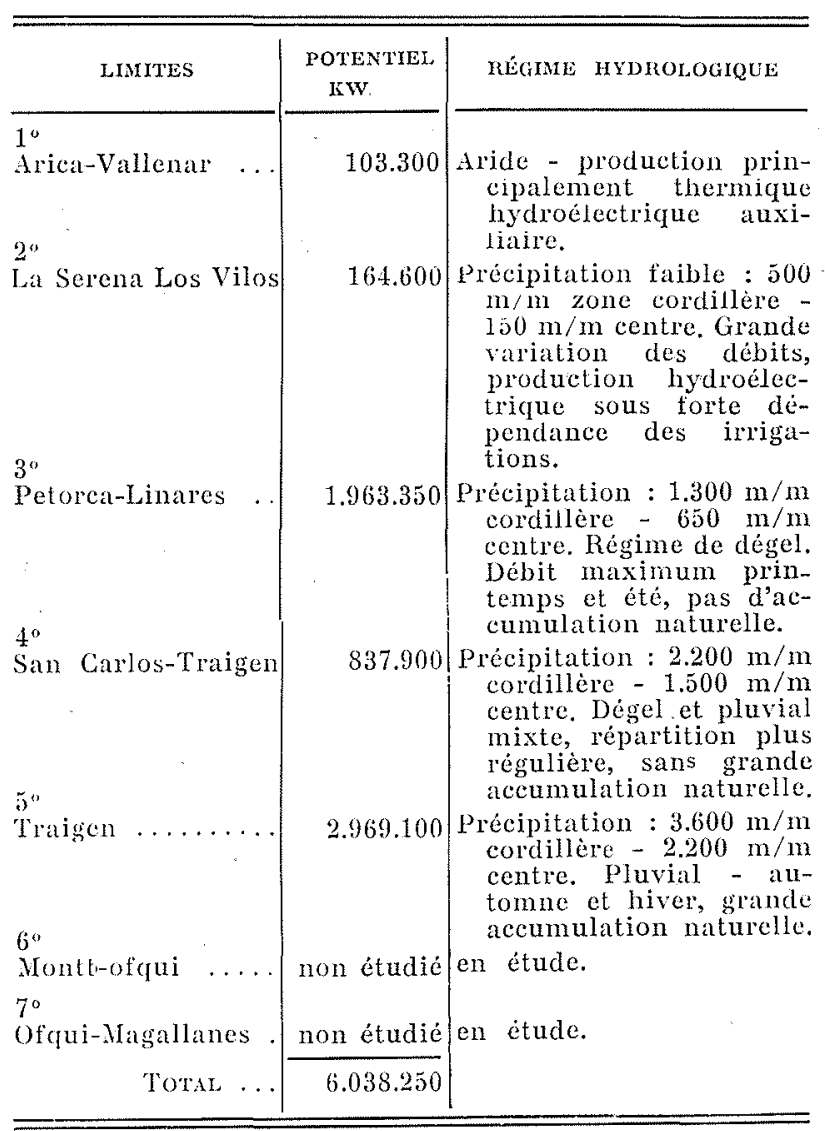

\section{PROGRAMILE DE REALISATTON}

La réalisation du plan a été prévue en trois grandes étapes chacune tenant compte, dans la plus grande mesure possible, du développement des besoins de courant déterminés d'avance.

La première étape, qui s'étend sur une durée de 18 ans, complée à partir de 1939, comprend la construction d'un premier lot de centrales avec interconnexion interne de chaque région. Ce premier équipement ne présente qu'une faible part du potentiel total. Il est limité à 1.000 .000 kw environ, et l'on a fixé l'état d'avancement en 1958 , comme suit, en fonction du potentiel total : en exploitation $16 \%$, en construction $3 \%$, en étude $15 \%$. 
Voici quelques-unes des centrales hydroélectriques les plus importantes, dont la construction est prévue pendant la première étape :

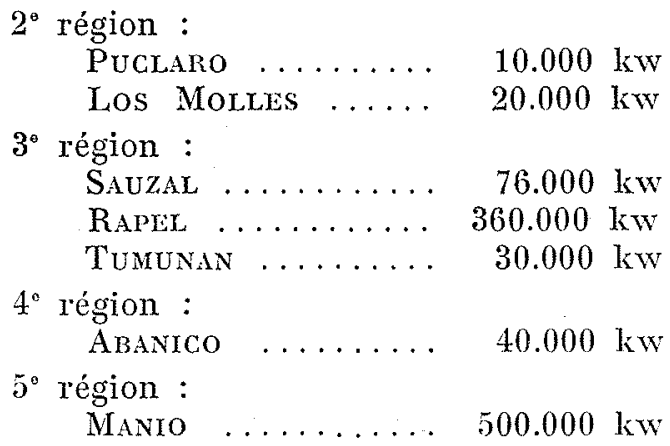

La seconde étape est destinée, d'une part, à la continuation de la réalisation de centrales et à l'interconnexion partielle des diverses régions autant qu'un échange d'énergie de puissance relativement faible, entre centrales de diverses régions, se justifie.

La troisième étape est destinée à l'achèvement de l'équipement et à l'interconnexion totale du pays, pour permettre la distribution, par dispatching unique, dans l'ensemble du pays.

\section{FINANCEMENT}

Le financement du plan d'électrification fait l'objet d'un plan chronologique tenant compte de l'augmentation des besoins, c'est-à-dire des possibilités de vente et du coût des équipements réalisés progressivement en admettant que la fourniture des machines hydrauliques et du matériel électrique ne soit pas entravée par des difficultés d'ordre financier et technique.

Pour les 18 premières années, correspondant à la première étape, les inversions totales sont de 2.399.166.000 pesos chilenos, dont 1.674.380 mille pesos d'apports et 724.777 .000 pesos provenant des ventes probables de courant pendant cette même période. Les apports sont particulièrement élevés pendant les 6 premières années et l'Etat compte bien les couvrir en grande partie, par des crédits consentis par les constructeur's étrangers. 